\title{
Quantifying Habitual Levels of Physical Activity According to Impact in Older People: Accelerometry Protocol for the VIBE Study
}

\author{
Kevin C. Deere, Kimberly Hannam, Jessica Coulson, Alex Ireland, Jamie S. McPhee, \\ Charlotte Moss, Mark H. Edwards, Elaine Dennison, Cyrus Cooper, Adrian Sayers, \\ Matthijs Lipperts, Bernd Grimm, and Jon H. Tobias
}

\begin{abstract}
Physical activity (PA) may need to produce high impacts to be osteogenic. The aim of this study was to identify threshold(s) for defining high impact PA for future analyses in the VIBE (Vertical Impact and Bone in the Elderly) study, based on home recordings with triaxial accelerometers. Recordings were obtained from 19 Master Athlete Cohort (MAC; mean 67.6 years) and 15 Hertfordshire Cohort Study (HCS; mean 77.7 years) participants. Data cleaning protocols were developed to exclude artifacts. Accelerations expressed in $g$ units were categorized into three bands selected from the distribution of positive Y-axis peak accelerations. Data were available for 6.6 and 4.4 days from MAC and HCS participants respectively, with approximately $14 \mathrm{hr}$ recording daily. Three-fold more $0.5-1.0 \mathrm{~g}$ impacts were observed in MAC versus HCS, 20 -fold more 1.0-1.5g impacts, and 140-fold more impacts $\geq 1.5 \mathrm{~g}$. Our analysis protocol successfully distinguishes PA levels in active and sedentary older individuals.
\end{abstract}

Keywords: peak $g$, accelerometry, VIBE

Accelerometry is widely used to provide an objective measure of physical activity (PA). In the case of Actigraph devices, which are most commonly employed, the raw signal is processed into counts per minute, which have previously been calibrated in terms of energy consumption (Mattocks et al., 2007) and provide valuable insights into relationships between PA and health outcomes such as obesity (Ness et al., 2007). It may also be possible to use accelerometry to evaluate effects of PA on bone by focusing on vertical accelerations, which reflect impacts resulting from weight bearing activity to which the skeleton preferentially responds, since the latter is influenced by skeletal deformation (quantified as 'strain') (Rubin \& Lanyon, 1985). However, high-impact events may be very brief, and removed by conventional processing algorithms. Hence, accelerometers which enable the detection of isolated high impacts have been developed to explore relationships between high impact PA and hip bone mineral density (BMD) in adolescents (Deere, Sayers, Rittweger, \& Tobias, 2012) and premenopausal women (Vainionpaa, Korpelainen, Leppaluoto, \& Jamsa, 2005). These studies have confirmed the importance of high impacts in maintaining hip BMD.

One of the challenges in applying an equivalent approach to older individuals is that high-impact activities such as running and jumping are rarely undertaken in this age group. For example, whereas approximately $4 g$ was used as a cut off indicating high impact in adolescents and premenopausal women above, our pilot

Deere, Hannam, Sayers, and Tobias are with the Musculoskeletal Research Unit, School of Clinical Sciences, University of Bristol, Bristol, UK. Coulson, Ireland, and McPhee are with the School of Healthcare Science, Manchester Metropolitan University, Manchester, UK. Moss, Edwards, Dennison, and Cooper are with the MRC Lifecourse Epidemiology Unit, University of Southampton, Southampton, UK. Lipperts and Grimm are with Atrium Medical Centre, Heerlen, Netherlands. Address author correspondence to Jon H. Tobias at Jon.Tobias@bristol.ac.uk. studies indicate that older individuals rarely experience vertical impacts $>2 g$ (Tobias et al., 2014). Nevertheless, it would seem likely that effects on bone outcomes depend on $g$ level, even within $g$ ranges related to lower impact activities. We aimed to investigate this question in the Vertical Impact and Bone in the Elderly (VIBE) study by partitioning vertical impacts obtained from seven-day accelerometer recordings, according to level of impact. Different population-based cohorts of older individuals are involved, namely the National Survey of Health and Development (NSHD) (Kuh et al., 2011), the Hertfordshire Cohort study (HCS) (Syddall et al., 2005), the Cohort of Skeletal Health in Bristol and Avon (COSHIBA) (Clark et al., 2012), as well as the newly recruited Master Athletes Cohort (MAC).

In VIBE, participants are asked to wear a triaxial GCDC accelerometer (Gulf Coast Data Concepts, LLC, Waveland, MS), which records the accelerometry signal in a comma separated values (CSV) format in a time-dependent manner. In a recent pilot study undertaken in older participants attending an aerobics exercise class, we confirmed that the magnitude of acceleration peaks as recorded by this device accurately reflects the level of impact activity undertaken (Hannam, Deere, Worral, Harltey, \& Tobias, 2016). Herein, we present our protocol for processing seven-day accelerometry recordings obtained using the GCDC accelerometer, which was developed based on 40 initial recordings obtained from HCS and MAC, which were assumed to be broadly representative of the VIBE study as a whole. In particular, we aimed to identify threshold(s) for defining high-impact PA for future analyses in VIBE. We also describe how accelerometry data are cleaned to exclude artifacts, reflecting events such as jarring or dropping the monitor, which may complicate interpretation of results particularly if only very few accelerations related to high impacts are recorded. In addition, to determine if our method is suitable for application to the wider VIBE study, we aimed to evaluate feasibility, based on the length of time taken to process each individual's recording, and face validity, based on the ability to detect the considerably higher levels of habitual PA in MAC as compared with HCS participants. 


\section{Methods}

\section{Participants}

The analysis protocol was developed based on the first 40 VIBE participants (20 from MAC and 20 from HCS) who returned accelerometers with recorded PA data. MAC comprises individuals across the United Kingdom > 60 years of age who have competed at regional level in sprint, middle, or long distance athletics in the past 12 months (target $n=300$ ). HCS recruited approximately 3,000 men and women born in Hertfordshire between 1931-1939 who were still residents there in 1998-2003 (Syddall et al., 2005). In addition to the accelerometry, all participants completed a questionnaire collecting individual demographic, health status, and activity data. Separate approvals were obtained from the National Research Ethics Service (NRES) for each cohort, and written informed consent was provided by all participants.

\section{Wearing the Accelerometer}

Participants received an activated GCDC $\times 16-1 \mathrm{c}$ triaxial accelerometer in the mail. The device, which has a built-in real-time clock, was fitted with a new lithium battery before being sent out. Participants also received an elasticated belt, detailed instructions for use, and a stamped addressed package for returning after use. We used a color coding system to ensure that devices were correctly positioned within the belt, which was available in different sizes to ensure this could be securely positioned over the right hip. Participants were asked to wear the monitor for seven consecutive days, and to complete a time sheet of when they put it on in the morning and took it off before going to bed, as well as an opportunity to state if the day had been a reflection of normal daily activity, along with a reason if not. Although the monitor has a maximum sampling frequency of $100 \mathrm{~Hz}$, data sampling rate was set at $50 \mathrm{~Hz}$ to ensure that battery life was sufficient to last the full seven-day recording period (use of $50 \mathrm{~Hz}$ should be sufficient to detect brief high-impact movements, based on the profile of vertical ground reaction forces during sprinting, which suggests that sampling at a frequency of $50 \mathrm{~Hz}$ will be sufficient to capture peak values) (Hunter, Marshall, $\&$ McNair, 2005). Deadband setting (i.e., the duration of inactivity beyond which the monitor turned itself off to conserve battery life) was $10 \mathrm{~s}$. The monitor was set to write 50,000 observations per CSV file.

\section{Accelerometry Data Cleaning}

Analyses were restricted to vertical (i.e., Y axis) accelerations. Individual CSV data were converted to a Stata data file (College Station, TX). The start and end of each day was determined by identification of nonwear through prolonged sequences of zero movement readings, cross-checked against time sheets. Sequences of zero readings lasting longer than 20 min were used to identify periods when the monitor was removed during the day, which were then excluded. Wearing of the device upside down was recognized by predominantly negative $\mathrm{Y}$ axis accelerations, in which case $g$ values were inverted providing this occurred infrequently.

A data cleaning protocol was developed to exclude movement artifacts. Data were removed $1 \mathrm{~min}$ at either side of the start and end of the day to exclude artifacts related to taking the monitor on and off. Data were also excluded 1 min at either side of periods when the monitor was removed during the day. In addition, isolated accelerations $>2 g$ occurring on a background of low activity were removed, on the basis that these are likely to result from artifacts such as dropping or knocking the monitor, rather than high-impact PA during which multiple accelerations are recorded even for very brief activities. A given day was excluded if less than $10 \mathrm{hr}$ of valid recording time was obtained, to ensure that a given day's recording was representative of the total amount of PA undertaken. Participants were excluded who failed to meet the minimum requirement of three days with a minimum of $10 \mathrm{hr}$ recording, as in our previous study based on adolescents (Deere et al., 2012).

\section{Acceleration Counts}

Y-axis acceleration peaks were identified based on accelerations which were higher than the preceding and subsequent reading (in the absence of any smoothing algorithm, a single movement frequently comprised multiple acceleration peaks). The number of positive Y-axis acceleration peaks was recorded within different $g$ bands, reflecting impacts from ground reaction forces of different magnitude. The number of acceleration peaks within 14 prespecified $g$ bands (i.e., $0.5 \leq g<1.0 ; 1.0 \leq g<1.5 ; 1.5 \leq g<2.0 ; 2.0 \leq g$ $<2.5 ; 2.5 \leq g<3.0 ; 3.0 \leq g<3.5 ; 3.5 \leq g<4.0 ; 4.0 \leq g<4.5 ; 4.5$ $\leq g<5.0 ; 5.0 \leq g<6.0 ; 6.0 \leq g<7.0 ; 7.0 \leq g<8.0 ; 8.0 \leq g<9.0$; $g \geq 9.0$ ) was normalized for wear time by expression as number of counts per week (movements $\leq 0.5 g$ were excluded on the basis that these represent sedentary activity; $g$ values represent $g$ units over and above $1 g$ resulting from the earth's gravitational force). Results were then grouped into three distinct impact bands to facilitate subsequent analysis with respect to bone outcomes (Deere et al., 2012). A similar range, i.e., 0.5-1.0g, was used to denote low impact PA as previously. In light of findings from our previous pilot study that older participants attending aerobics classes do not experience accelerations beyond $2.1 \mathrm{~g}$ (Tobias et al., 2014), $g$ cut-offs for high impact were based on the distribution of counts observed. Summary statistics were obtained separately for MAC and HCS, expressed as median and 25 th and 75 th centiles.

\section{Results}

\section{Participant Characteristics}

In total, $19 \mathrm{MAC}$ and $15 \mathrm{HCS}$ study participants met our minimum criteria of $10 \mathrm{hr}$ valid recording for three days and formed the basis of the present analysis, characteristics of whom are shown in Table 1 . Of those, $76 \%$ of participants in this initial dataset were male. HCS participants were approximately nine years older than MAC participants, and approximately $10 \mathrm{~kg}$ (i.e., 15\%) heavier. All included participants reported fair to very good health; $74 \%$ of MAC participants reported to be in very good health compared with $29 \%$ of HCS participants. Hours of reported physical activity from the past week was almost double in the MAC (14.2 hr) participants compared with HCS participants (7.5 hr).

\section{Duration of Accelerometer Recording}

According to timesheets, each of the 34 participants included in this analysis wore the monitor for seven days, giving 238 days of recording. In spite of our use of elasticated belts that were available in different sizes, in the comments section two participants noted they found wearing the belt uncomfortable, and one participant reported that the monitor fell out of the belt on one occasion during a run. Thirty-five days were subsequently excluded as they did not reach the minimum of $10 \mathrm{hr}$ of valid recording. This was due to a combination of monitors not being worn for sufficiently long, batteries running out before the end of the week, and exclusion of nonwear time 
Table 1 Participant Characteristics

\begin{tabular}{lcc}
\hline & \multicolumn{2}{c}{ Cohort } \\
\cline { 2 - 3 } Characteristic & MAC $(\boldsymbol{n}=19)$ & HCS $(\boldsymbol{n}=15)$ \\
\hline Age & $67.6(60.1,76.6)$ & $77.7(75.2,81.8)$ \\
Male & 13 & 13 \\
Female & 6 & 2 \\
Height $(\mathrm{m})$ & $1.70(1.52,1.91)$ & $1.73(1.57,1.85)$ \\
Weight $(\mathrm{kg})$ & $63.4(46.3,86.6)$ & $73.3(57.2,91.2)$ \\
Body mass index $\left(\mathrm{kg} / \mathrm{m}^{2}\right)$ & $21.7(17.5,26.7)$ & $24.5(18.3,29.8)$ \\
Health status (self-reported) & & $4(28.6 \%)$ \\
Very good & $14(73.7 \%)$ & $5(35.7 \%)$ \\
Good & $1(5.3 \%)$ & $5(35.7 \%)$ \\
Fair & 0 & 0 \\
Poor & 0 & 0 \\
Very poor & $14.2(4,31)$ & $7.5(0,14)$ \\
Hours of PA in last 7 days (self-reported) & & \\
\hline Abbreviations: PA = physical activity; MAC = Master Athletes Cohort; HCS = Hertfordshire Cohort study. \\
$\begin{array}{l}\text { Note. Table shows characteristics of MAC and HCS participants included in the analysis. Results show mean and } \\
\text { range. }\end{array}$
\end{tabular}

during data processing. Data cleaning initially decreased recording time by a mean of 3 min and $5 \mathrm{~s}$ per participant, but in the case of two HCS participants this led to the duration of three recording days falling below the $10 \mathrm{hr}$ valid recording threshold, leading to the whole of these days being removed. A further 12 days were lost due to the monitor being flipped over on multiple occasions, making it hard to identify positive y-axis accelerations. In total, 191 days (i.e., $80 \%$ of recording days) were included in subsequent analyses. The minimum threshold of $10 \mathrm{hr}$ valid recording was achieved for a mean of 6.6 days and 4.4 days in MAC and HCS participants respectively. Duration of valid recording per valid day was similar in both groups (i.e., approximately $14 \mathrm{hr}$ and $18 \mathrm{~min}$ ).

\section{Acceleration Peaks}

Data cleaning led to removal of a small proportion of acceleration peaks, with the exception of the two HCS participants in whom duration of three recording days fell below the $10 \mathrm{hr}$ recording threshold. In MAC participants, a total of 685 peaks between $0.5-1.0 \mathrm{~g}$ were removed, 141 peaks between $1.0-2.0 \mathrm{~g}$, and $31 \geq 2.0 \mathrm{~g}$. In HCS, 8,568 peaks were removed between $0.5-1.0 \mathrm{~g}, 494$ between $1.0-2.0 \mathrm{~g}$, and $29 \geq 2.0 \mathrm{~g}$. In total, data processing and cleaning up until the point of generating a final number of acceleration peaks within different bands took approximately $90 \mathrm{~min}$ per individual.

Table 2 shows the median number of acceleration peaks per week in MAC and HCS cohorts, for each of the 14 prespecified $g$ bands, following adjustment for number of valid days recording. As expected, median acceleration peaks decreased with each successive increment in $g$ band; this change was considerably more marked in HCS compared with MAC. Median peaks per week was approximately three times higher in MAC compared with HCS for 0.5-1.0 $g$ acceleration peaks, 20-fold higher for 1.0-1.5 $g$ peaks, and 60 -fold higher for $1.5-2.0 \mathrm{~g}$ peaks. Substantial numbers of accelerations were seen in MAC above $2.0 \mathrm{~g}$. In contrast, very few higher acceleration peaks were observed in HCS, with a median of 12 counts per week in the range $2.0-2.5 g$ and virtually no accelerations beyond this.
Subsequently, we aimed to group counts into three distinct bands to facilitate subsequent analyses. Low impacts were identified based on acceleration peaks in the lowest band (i.e., 0.5-1.0g). Based on the distribution of counts shown in Table 2, 'high' impact was defined using two different thresholds, i.e., $\geq 1.5 \mathrm{~g}$ (Table 3A; medium counts $1.0 \leq g<1.5 g$ ) and $\geq 2.0 \mathrm{~g}$ (Table $3 \mathrm{~B}$; medium counts $1.0 \leq g<2.0 \mathrm{~g}$ ). Using the lower (i.e., $1.5 \mathrm{~g}$ ) threshold, the number of medium and high impact peaks in MAC were $40 \%$ and $54 \%$ those of low impacts respectively, based on values for median number of acceleration peaks per week. The equivalent percentage in HCS was $5 \%$ and $1 \%$ for medium and high impacts respectively. There were approximately 140 times more high impact peaks in MAC compared with HCS. Based on the higher (i.e., 2.0g) threshold, medium and high impact peaks were $67 \%$ and $27 \%$ those of low impact peaks in MAC, and $7 \%$ and $0.2 \%$ those of low impact peaks in HCS. There were approximately 400-fold more high impact peaks in MAC compared with HCS.

\section{Discussion}

We report findings from our study intended to develop the accelerometry protocol in VIBE. Our results support wider application of this protocol to VIBE, enabling us to examine the overall study goals, namely characterization of habitual levels of high impact PA and how this relates to bone health and that of other systems. For example, application of the Stata code which we developed for data processing and cleaning takes approximately $90 \mathrm{~min}$ to run per participant, making it feasible to analyze data from the 2,000 participants which we anticipate collecting over an 18-month period. In addition, our method appears to have face validity, given our observation that higher intensity vertical impacts were considerably more frequent in MAC compared with HCS.

The accelerometry protocol in VIBE appeared to be acceptable to participants as judged by the relatively long duration of recording time obtained, and the fact that valid data were obtained for the majority of available days. Some of the reasons why valid 
Table 2 Accelerometer Counts (14 Bands)

\begin{tabular}{|c|c|c|c|c|c|c|}
\hline \multirow[b]{2}{*}{ G-band } & \multicolumn{3}{|c|}{ MAC $(n=19)$} & \multicolumn{3}{|c|}{ HCS $(n=15)$} \\
\hline & Median & 25th & 75th & Median & 25th & 75th \\
\hline $0.5<\mathrm{g}<1.0^{1}$ & 32,979 & 20,719 & 57,685 & 10,022 & 5,929 & 25,401 \\
\hline $1.0 \leq \mathrm{g}<1.5^{2}$ & 13,086 & 9,072 & 21,301 & 548 & 163 & 18,535 \\
\hline $1.5 \leq \mathrm{g}<2.0^{3}$ & 6,384 & 3,476 & 15,688 & 107 & 14 & 210 \\
\hline $2.0 \leq \mathrm{g}<2.5^{4}$ & 5,347 & 931 & 8,339 & 12 & 2 & 42 \\
\hline $2.5 \leq \mathrm{g}<3.0^{4}$ & 2,053 & 432 & 5,098 & 2 & 1 & 10 \\
\hline $3.0 \leq \mathrm{g}<3.5^{4}$ & 425 & 200 & 2,363 & 2 & 0 & 5 \\
\hline $3.5 \leq \mathrm{g}<4.0^{4}$ & 120 & 61 & 674 & 0 & 0 & 0 \\
\hline $4.0 \leq \mathrm{g}<4.5^{4}$ & 41 & 19 & 172 & 0 & 0 & 0 \\
\hline $4.5 \leq \mathrm{g}<5.0^{4}$ & 13 & 4 & 56 & 0 & 0 & 0 \\
\hline $5.0 \leq \mathrm{g}<6.0^{4}$ & 7 & 1 & 29 & 0 & 0 & 0 \\
\hline $6.0 \leq \mathrm{g}<7.0^{4}$ & 1 & 0 & 2 & 0 & 0 & 0 \\
\hline $7.0 \leq \mathrm{g}<8.0^{4}$ & 0 & 0 & 1 & 0 & 0 & 0 \\
\hline $8.0 \leq \mathrm{g}<9.0^{4}$ & 0 & 0 & 0 & 0 & 0 & 0 \\
\hline $\mathrm{g} \geq 9.0^{4}$ & 0 & 0 & 0 & 0 & 0 & 0 \\
\hline
\end{tabular}

Abbreviations: $\mathrm{MAC}=$ Master Athletes Cohort $\mathrm{HCS}=$ Hertfordshire Cohort study.

Note. Number of acceleration peaks per week within 14 different acceleration bands, in MAC and HCS participants. Data are shown as median and 25th and 75th centiles. ${ }^{1}$ Low, ${ }^{2}$ medium, and ${ }^{4}$ high impact bands (see Table 3 ). ${ }^{3}$ Medium or high impact according to which threshold was selected.

Table 3 Accelerometer Counts (Three Bands)

\begin{tabular}{|c|c|c|c|c|}
\hline \multirow[b]{2}{*}{ G-band } & \multicolumn{2}{|c|}{ MAC $(n=19)$} & \multicolumn{2}{|c|}{$\operatorname{HCS}(n=15)$} \\
\hline & Median & 25th, 75th Centiles & Median & 25th, 75th Centiles \\
\hline \multicolumn{5}{|c|}{ (A) High-impact threshold $=1.5 \mathrm{~g}$} \\
\hline Low & 32,979 & 20,$719 ; 57,685$ & 10,022 & 5,$929 ; 25,401$ \\
\hline Medium & 13,086 & 9,$072 ; 21,301$ & 548 & $163 ; 1,853$ \\
\hline High & 17,669 & 5,$584 ; 28,210$ & 128 & $16 ; 259$ \\
\hline \multicolumn{5}{|c|}{ (B) High-impact threshold $=2.0 \mathrm{~g}$} \\
\hline Low & 32,979 & 20,$719 ; 57,685$ & 10,022 & 5,$929 ; 25,401$ \\
\hline Medium & 21,985 & 13,$429 ; 40,445$ & 674 & $182 ; 2,118$ \\
\hline High & 8,807 & 1,$659 ; 17,692$ & 21 & $3 ; 49$ \\
\hline
\end{tabular}

Abbreviations: $\mathrm{MAC}=$ Master Athletes Cohort $\mathrm{HCS}=$ Hertfordshire Cohort study.

Note. Number of acceleration peaks per week grouped into low, medium, and high impact bands, in MAC and HCS participants. (A) Low $0.5<g<1.0$; medium $1.0 \leq g<1.5 g$; high $g \geq 1.5$. (B) Low $0.5<g<1.0 g$; medium $1.0 \leq g<2.0 g$; high: $g \geq 2.0$. Data are shown as median and 25 th and 75 th centiles.

recording days were lost may be modifiable. For example, it may be possible to prevent the battery from running out by encouraging participants to start to wear the monitor as soon as they receive it. Instances of the monitor frequently flipping over may reflect inadequate participant instructions or poorly fitting elasticated belts, issues which have since been addressed for the ongoing study. The distribution of acceleration peaks within bands was as predicted, with a concentration of peaks within the lower $g$ bands, which was considerably more marked in HCS as compared with MAC; this is in keeping with the fact that MAC participants were an average of nine years younger than HCS participants and, unlike HCS, were selectively recruited as a consequence of their high levels of PA. In terms of our analysis protocol, the application of algorithms intended to remove artifacts only led to minor changes in recording duration and number of acceleration peaks.

We aimed to identify threshold(s) for defining high-impact PA for future analyses in VIBE. The ultimate aim is to use this information to identify target $g$ levels for improving skeletal health and that of other systems. Since these $g$ bands relate to specific activities (see Table 4), this approach is expected to provide the basis for public health interventions intended to increase exposure to specific levels of impact. Although activities such as running and jumping 
which generate high impacts are rarely undertaken in unselected populations of older people, high impacts may still be important for skeletal health in this age group despite their rarity. For example, a hopping intervention was recently found to increase hip BMD in older men with a mean 70 years of age (Allison, Folland, Rennie, Summers, \& Brooke-Wavell, 2013).

Activities like hopping which involve rapid upward movement of the center of mass are expected to generate relatively high levels of impact. Although the range of $g$ values achieved by different high-impact activities in older adult populations remains to be defined, it is likely to exceed $2.0 \mathrm{~g}$, which is the highest threshold that could be applied to the dataset obtained from HCS participants since virtually no acceleration peaks were detected beyond $2.5 \mathrm{~g}$. Also, $2.0 \mathrm{~g}$ is considerably lower than the $4.0 \mathrm{~g}$ threshold used to define high-impact PA in our previous studies based on ALSPAC adolescents (Deere et al., 2012). Theoretically, a lower $g$ may produce an equivalent strain in older individuals compared with a higher $g$ in younger individuals, due to age-related declines in bone strength. Therefore, the level of force required to elicit a significant bone response is likely to be lower in older individuals, in which case a lower $g$ threshold to identify high impacts in this group may be appropriate.

Using a higher threshold to define high-impact PA leads to a greater specificity and is expected to result in a greater effect size when examining relationships with outcomes such as bone health. However, this needs to be balanced against both reduced sensitivity due to failure to include certain types of osteogenic activity, and lack of power due to insufficient events being recorded. Use of a $2.0 \mathrm{~g}$ threshold may be problematic in future analyses since very few impacts were recorded within this range in HCS, with a median of only 21 acceleration peaks per week observed in our initial subsample. Although this limitation did not apply to MAC, which was recruited on the basis of high levels of PA, it is also likely to be the case in other population based cohorts which contribute to VIBE, namely NSHD and COSHIBA. Hence, it may be necessary to lower the upper threshold to ensure sufficient power. For this reason we also examined use of a $1.5 \mathrm{~g}$ threshold to define high-impact PA, which resulted in approximately six times more high impact peaks being detected in HCS as compared with use of the $2.0 \mathrm{~g}$ threshold. Use of a $1.5 \mathrm{~g}$ threshold to define high impact in older populations is also in line with results of our pilot study in which older participants undertaking an aerobics class accrued counts within the range $1.5-2.0 \mathrm{~g}$, whereas virtually no counts were seen above this (Tobias et al., 2014).

\section{Table 4 Activities Associated with Different Bands}

\begin{tabular}{ll}
\hline G Band & Activity \\
\hline $0.5-1.0 \mathrm{~g}$ & Walking ${ }^{1,2}$ \\
$1.0-1.5 \mathrm{~g}$ & Stepping $^{1,2}$ \\
$1.5-2.0 \mathrm{~g}$ & Half jacks $^{1}$ \\
$2.0-3.0 \mathrm{~g}$ & Lateral jumping $^{2}$ \\
$3.0-4.0 \mathrm{~g}$ & Jogging $^{2}$ \\
$4.0-5.0 \mathrm{~g}$ & Running, jumping $^{2}$ \\
$>5.0 \mathrm{~g}$ & Drop jumps $^{2}$ \\
\hline
\end{tabular}

Note. Table shows ranges of peak $g$ typically achieved with different activities based on ${ }^{1} 20$ women (mean age 67 years) attending a supervised aerobics class (Tobias et al., 2014) and ${ }^{2} 10$ women performing exercises under supervision (aged 20-58) (Vainionpaa et al., 2006).
In terms of generalizability of our method, it should be straightforward to apply the Stata code which we derived as part of this study to other equivalent datasets. The main proviso is that the analysis protocol which we have developed is specific to the accelerometer settings used in VIBE, and minor modifications would be needed if different sampling frequency, deadband duration, or number of observations per CSV file is selected.

\section{Limitations}

One limitation is that the protocol was developed based on a subset of VIBE participants. However, we reasoned that a convenience sample drawn from these two cohorts would generate accelerometry data that was broadly representative of VIBE as a whole, given that HCS participants are among the oldest of VIBE subjects, whereas MAC are the youngest and most active. A limitation of our analysis method is that acceleration peaks were identified on the basis of a $g$ value which was greater than the one immediately before and afterward; in the absence of a smoothing algorithm, any one movement may have contributed several values for peak $g$. Our observation that MAC had far greater numbers of impacts than HCS provides face validity for our assumption that the number of acceleration peaks identified in this way reflects the number of impacts actually experienced. Furthermore, in a recent pilot study in which the same monitors were worn by individuals (mean age 69 years) during an aerobics class (Hannam et al., 2016), in the high intensity running component, a close relationship was observed between number of movements (based on manual inspection of acceleration profiles during the activity) and acceleration peaks (number of movements $=47.8 \pm 11.5$, number of acceleration peaks $=50.5 \pm 13.2[n=$ 39 , mean $\pm S D], r=.82$ ). Further validation of our accelerometer protocol is expected to follow after wider application in VIBE, in which we plan to relate accelerometer data as described here to questionnaire-based PA assessments, and clinical measures known to predict PA levels such as the short physical performance battery.

\section{Conclusion}

This paper has focused on the principle accelerometry variables to be derived in the VIBE cohorts for use in subsequent analyses relating high-impact PA to bone and other health-related outcomes. As such, the number of high-impact acceleration peaks is expected to be our primary accelerometry variable of interest, though whether it is more appropriate to use the $1.5 \mathrm{~g}$ or $2.0 \mathrm{~g}$ threshold to define these remains to be established. In future studies, we aim to examine the feasibility of extracting additional PA characteristics from the dense raw data collected with the GCDC accelerometers. For example, we are keen to develop processing algorithms which detect the entire acceleration profile for each movement, enabling a single value for peak $g$ to be derived. In addition, it may be feasible to quantify specific activities such as walking based on recognition of movement patterns, and to identify lower limb pathology based on the identification of movement asymmetry (Yoneyama, 2015). Finally, it may be possible to gain a more accurate reflection of bone strain, which is influenced by movements in all planes, by deriving measures of acceleration vectors from all three axes.

\section{Acknowledgments}

We are very grateful to the MAC and HCS participants who enrolled in this study. The Hertfordshire Cohort Study was supported by the Medical Research Council (MRC) of Great Britain, Arthritis Research UK, and the International Osteoporosis Foundation. VIBE is funded by the Medical 
Research Council grant ref MR/K024973/1, for which we acknowledge the contribution of the other co-applicants, namely Prof George Davey-Smith (University of Bristol), Prof Joern Rittweger (German Aerospace Centre), Prof Seif Shaeen (Queen Mary, University of London), Prof Avan Aihie Sayer (University of Southampton), Prof Ken Fox (University of Bristol), Prof Diana Kuh (MRC Unit for Lifelong Health and Ageing), Prof Marcus Richards (MRC Unit for Lifelong Health and Ageing), Dr Rebecca Hardy (MRC Unit for Lifelong Health and Ageing), Dr Rachel Cooper (MRC Unit for Lifelong Health and Ageing), Dr Kate Ward (MRC Human Nutrition Research Unit), and Dr Soren Brage (MRC Epidemiology Unit).

\section{References}

Allison, S.J., Folland, J.P., Rennie, W.J., Summers, G.D., \& Brooke-Wavell, K. (2013). High impact exercise increased femoral neck bone mineral density in older men: a randomised unilateral intervention. Bone, 53(2), 321-328. PubMed doi:10.1016/j.bone.2012.12.045

Clark, E.M., Gould, V., Morrison, L., Ades, A.E., Dieppe, P., \& Tobias, J.H. (2012). Randomized controlled trial of a primary care-based screening program to identify older women with prevalent osteoporotic vertebral fractures: Cohort for Skeletal Health in Bristol and Avon (COSHIBA). Journal of Bone and Mineral Research, 27(3), 664-671. PubMed doi:10.1002/jbmr.1478

Deere, K., Sayers, A., Rittweger, J., \& Tobias, J. (2012). Habitual levels of high, but not moderate or low, impact activity are positively related to hip BMD and geometry: Results from a population-based study of adolescents. Journal of Bone and Mineral Research, 27(9), 1887-1895. PubMed doi:10.1002/jbmr.1631

Hannam, K., Deere, K., Worral, S., Hartley, A., Tobias J. H. (2016) Characterization of vertical accelerations experienced by older people attending an aerobics class designed to produce high impacts. Journal of Aging and Physical Activity, 24(2). doi: 10.1123/japa.2015-0060.

Hunter, J.P., Marshall, R.N., \& McNair, P.J. (2005). Relationships between ground reaction force impulse and kinematics of sprint-running acceleration. Journal of Applied Biomechanics, 21, 31-43. PubMed
Kuh, D., Pierce, M., Adams, J., Deanfield, J., Ekelund, U., Friberg, P., \& Hardy, R. (2011). Cohort profile: updating the cohort profile for the MRC National Survey of Health and Development: a new clinic-based data collection for ageing research. International Journal of Epidemiology, 40(1), e1-e9. PubMed doi:10.1093/ije/dyq231

Mattocks, C., Leary, S., Ness, A., Deere, K., Saunders, J., Tilling, K., \& Riddoch, C. (2007). Calibration of an accelerometer during free-living activities in children. International Journal of Pediatric Obesity; IJPO, 2, 218-226. PubMed

Ness, A.R., Leary, S.D., Mattocks, C., Blair, S.N., Reilly, J.J., Wells, J., \& Riddoch, C. (2007). Objectively measured physical activity and fat mass in a large cohort of children. PLoS Medicine, 4(3), e97. PubMed doi:10.1371/journal.pmed.0040097

Rubin, C.T., \& Lanyon, L.E. (1985). Regulation of bone mass by mechanical strain magnitude. Calcified Tissue International, 37, 411-417. PubMed doi:10.1007/BF02553711

Syddall, H.E., Aihie Sayer, A., Dennison, E.M., Martin, H.J., Barker, D.J., \& Cooper, C. (2005). Cohort profile: the Hertfordshire cohort study. International Journal of Epidemiology, 34(6), 1234-1242. PubMed doi:10.1093/ije/dyi127

Tobias, J.H., Gould, V., Brunton, L., Deere, K., Rittweger, J., Lipperts, M., \& Grimm, B. (2014). Physical activity and bone: may the force be with you. Frontiers in Endocrinology, 5, 20. PubMed

Vainionpaa, A., Korpelainen, R., Leppaluoto, J., \& Jamsa, T. (2005). Effects of high-impact exercise on bone mineral density: a randomized controlled trial in premenopausal women. Osteoporosis International, 16(2), 191-197. PubMed doi:10.1007/s00198-004-1659-5

Vainionpaa, A., Korpelainen, R., Vihriala, E., Rinta-Paavola, A., Leppaluoto, J., \& Jamsa, T. (2006). Intensity of exercise is associated with bone density change in premenopausal women. Osteoporosis International, 17(3), 455-463. PubMed doi:10.1007/s00198-005-0005-x

Yoneyama, M. (2015). Visualising gait symmetry/asymmetry from acceleration data. Computer Methods in Biomechanics and Biomedical Engineering, 18(9), 923-930. PubMed doi:10.1080/10255842.201 3.856892 